\title{
Subclinical leaflet thrombosis is associated with impaired reverse remodelling after transcatheter aortic valve implantation
}

\section{Bálint Szilveszter (1) ${ }^{1}$, Daniel Oren ${ }^{1}$, Levente Molnár ${ }^{1}$, Astrid Apor ${ }^{1}$, Anikó I. Nagy', Andrea Molnár ${ }^{1}$, Borbála Vattay ${ }^{1}$, Márton Kolossváry ${ }^{1}$, Júlia Karády ${ }^{1}$, Andrea Bartykowszki ${ }^{1}$, Ádám L. Jermendy ${ }^{1}$, Ferenc I. Suhai ${ }^{1}$, Alexisz Panajotu ${ }^{1}$, Pál Maurovich-Horvat $\mathbb{B}^{1,2 *, \dagger}$, and Béla Merkely ${ }^{1, \dagger}$}

\author{
${ }^{1}$ MTA-SE Cardiovascular Imaging Research Group, Heart and Vascular Center, Semmelweis University, Városmajor Street, 1122 Budapest, Hungary; and ${ }^{2}$ Medical Imaging \\ Center, Semmelweis University, Üllöi Street, 1082 Budapest, Hungary
}

Received 10 April 2019; editorial decision 19 September 2019; accepted 24 October 2019; online publish-ahead-of-print 30 October 2019

Aims

Cardiac CT is increasingly applied for planning and follow-up of transcatheter aortic valve implantation (TAVI). However, there are no data available on reverse remodelling after TAVI assessed by CT. Therefore, we aimed to evaluate the predictors and the prognostic value of left ventricular (LV) reverse remodelling following TAVI using CT angiography.

Methods

and results

We investigated 117 patients with severe, symptomatic aortic stenosis (AS) who underwent CT scanning before and after TAVI procedure with a mean follow-up time of 2.6 years after TAVI. We found a significant reduction in LV mass (LVM) and LVM indexed to body surface area comparing pre- vs. post-TAVI images: $180.5 \pm 53.0$ vs. $137.1 \pm 44.8 \mathrm{~g}$ and $99.7 \pm 25.4$ vs. $75.4 \pm 19.9 \mathrm{~g} / \mathrm{m}^{2}$, respectively, both $P<0.001$. Subclinical leaflet thrombosis (SLT) was detected in $25.6 \%$ (30/117) patients. More than 20\% reduction in LVM was defined as reverse remodelling and was detected in $62.4 \%$ (73/117) of the patients. SLT, change in mean pressure gradient on echocardiography and prior myocardial infarction was independently associated with LV reverse remodelling after adjusting for age, gender, and traditional risk factors (hypertension, body mass index, diabetes mellitus, and hyperlipidaemia): $O R=0.27$, $P=0.022$ for $S L T$ and $O R=0.22, P=0.006$ for prior myocardial infarction, $O R=1.51, P=0.004$ for $10 \mathrm{mmHg}$ change in mean pressure gradient. Reverse remodelling was independently associated with favourable outcomes $(H R=0.23 ; P=0.019)$.

Conclusion

TAVI resulted in a significant LVM regression on CT. The presence of SLT showed an inverse association with LV reverse remodelling and thus it may hinder the beneficial LV structural changes. Reverse remodelling was associated with improved long-term prognosis.

Keywords

left ventricular reverse remodelling - transcatheter aortic valve implantation $\bullet$ CT angiography $\bullet$ subclinical leaflet thrombosis

\section{Introduction}

Aortic stenosis (AS) is the most common valvular disease in industrialized countries. ${ }^{1,2}$ It leads to chronic pressure overload of the left ventricle (LV) resulting in adaptational adverse remodelling. ${ }^{3}$ Left ventricular hypertrophy is a hallmark feature of AS and a strong predictor of mortality, characterized by both cellular and extracellular expansion of the myocardial tissue. ${ }^{4}$ Aortic valve replacement (AVR) is the only definitive therapeutic option for patients with severe, symptomatic AS. Transcatheter aortic valve implantation (TAVI) has

\footnotetext{
* Corresponding author. Tel: +36-20-666-3857; Fax: +36-1-458-6842. E-mail: maurovich.horvat@gmail.com

† These authors contributed equally to this work.

Published on behalf of the European Society of Cardiology. All rights reserved. @ The Author(s) 2019. For permissions, please email: journals.permissions@oup.com.
} 
emerged as a safe and effective alternative to surgical AVR in patients with intermediate to high surgical risk and severe, symptomatic AS., Furthermore, TAVI is rapidly expanding towards lower-risk patient populations and implantation rates are expected to further increase.

It has been suggested that postoperative LV mass (LVM) regression after AVR is associated with improved long-term outcomes. ${ }^{7}$ Therefore, recent studies aimed to define predictors of structural LV recovery after TAVI. To date, most studies evaluating valve function and reverse remodelling utilized 2D-echocardiography and reported a substantial reduction in LVM following TAVI or AVR. ${ }^{8-11}$ However, echocardiography has limited reproducibility for LVM assessment. ${ }^{12,13}$ In clinical practice, echocardiography is the first-line diagnostic test in case of suspected TAVI thrombosis; however, this modality has limited sensitivity to detect subtle changes in leaflet morphology that are readily detected on CT angiography (CTA). In many cases, not even transoesophageal echocardiography (TEE) can reliably directly visualize subclinical leaflet thrombosis (SLT) before overt haemodynamic consequences. Importantly, SLT using CT was detected in $\sim 0-40 \%$ of patients after TAVI procedure with currently controversial results on its clinical relevance. ${ }^{14-16}$ SLT has gained increased recognition as a possible risk factor for adverse cerebrovascular events ${ }^{14-16}$ and it could also inhibit beneficial reverse remodelling.

CTA provides simultaneous assessment of LV morphology and bioprosthetic valve changes, including SLT. ${ }^{16-19}$ We hypothesized that SLT might have a negative impact on reverse remodelling following TAVI. Therefore, we sought to define independent predictors (including SLT) of reverse remodelling and also aimed to investigate the prognostic value of $L V$ remodelling.

\section{Methods}

\section{Study population}

We analysed patients who underwent CTA for pre-TAVI planning and were assessed following TAVI to determine the presence or absence of SLT as part of the prospective RETORIC (Rule out Transcatheter Aortic Valve Thrombosis with Post Implantation Computed Tomography) trial. In brief, the prospective RETORIC study aims to define the predictors and clinical relevance of SLT in patients with severe, symptomatic AS who underwent TAVI. Patients who underwent TAVI procedure in our institute were included to evaluate bioprosthetic valve function and LV morphology. All patients received a self-expandable transcatheter aortic valve. Pre-TAVI imaging was performed to evaluate the aortic root anatomy and the access routes (iliofemoral and supraaortic arteries) to derive measurements for transcatheter heart valve sizing and eligibility for TAVI as a part of the routine clinical work-up. Follow-up CTA and echocardiography were performed on the same day to visualize leaflet thrombosis and to assess LV morphology at various time points following TAVI (average of 1.7 years). We excluded patients for whom CTA was contraindicated per institutional standard of care (history of severe and/or anaphylactic reaction, severe renal insufficiency defined as $\leq 30 \mathrm{~mL} / \mathrm{min} /$ $1.73 \mathrm{~m}^{2}$ ), or who had poor image quality for the evaluation of LVM (Figure 1).

The study was approved by the local and national ethics committees (SE-TUKEB234/2017; ENKK034489-004/2016/OTIG) and was registered on the Clinicaltrials.gov (NCT02826200) and was performed in accordance with the Helsinki declaration. Written informed consent was obtained from all patients.

\section{Image acquisition for TAVI planning and follow-up}

We performed retrospectively electrocardiogram-gated helical CTA of the aorta (from the level of thoracic inlet to the level of the femoral head) and the heart during a single breath-hold, in cranio-caudal direction for TAVI planning using a 256-slice CT scanner (Philips Healthcare, Best, The Netherlands, 270 ms rotation time, tube voltage of 100-120 kV). No oral beta-blocker was administered routinely prior to CTA imaging. We administered $75 \mathrm{~mL}$ iodinated contrast agent $(400 \mathrm{mg} / \mathrm{mL}$, lomeron 400 , Bracco Ltd; Milan, Italy) with $4.5 \mathrm{~mL} / \mathrm{s}$ flow rate and images were acquired with $1 \mathrm{~mm}$ slice thickness, $1 \mathrm{~mm}$ increment, and reconstructed using iterative reconstruction (iDose ${ }^{4}$ or IMR, Philips Healthcare, Cleveland, $\mathrm{OH}$, USA). Follow-up CT examinations were performed using retrospective gating with similar scan protocol, with shorter scan length covering the volume of the heart and the ascending aorta. At least 3 months has passed because the implantation prior to the follow-up CTA acquisition. CTA was performed in all patients - who did not have contraindication-irrespective of symptoms or echocardiographic results.

\section{CT image analysis}

LV myocardial mass was measured at the baseline and at the follow-up on CTA images using a semi-automated software in the end-systolic or end-diastolic phases (Philips Intellispace v6.0.4., Functional analysis tool, Best, The Netherlands). All measurements were performed in random order, blinded to the scan date and patient data. First, the software segmented the heart and created a short-axis stack through the LV. We adjusted the epi- and endocardial contours manually if needed. The software automatically calculated myocardial mass based on the left ventricular volumetric analysis. The calculated LVM value was indexed to the patients' body surface area. LV reverse remodelling was defined as a reduction of $>20 \%$ in calculated LVM and LVM index detected on postTAVI CT scans as compared with pre-TAVI CT scans.

The presence of SLT was evaluated by three radiologist with at least 5 years of experience in cardiac CT imaging. All readers were blinded to echocardiographic or clinical parameters and assessed SLT within the bioprosthetic valve in the end-diastolic phase. All cusps were evaluated separately for the presence of leaflet thrombosis and the number of involved cusps was recorded. The images were assessed by all three readers to achieve a consensus read. In long-axis view the thickness of each leaflet was measured in parallel and perpendicular axes to the frame. An average thickness of $>3 \mathrm{~mm}$ of the hypo-attenuated mass (based on both the parallel and the perpendicular axis measurements) was considered as leaflet thickening corresponding to SLTT. ${ }^{20}$ Representative images of SLT are shown in Figure 2.

\section{Preprocedural- and follow-up echocardiography}

All subjects underwent TTE using an EPIQ 7C system (Philips Medical System, Andover, MA, USA), equipped with X5-1 matrix transducer. The 2D grey-scale images were acquired over three heart cycles and analysed using QLab software (version 10.0 Philips Medical System, Andover, MA, USA) by one of four experienced echocardiographers, blinded to the CT data. Transaortic peak and mean pressure gradients were derived using the simplified Bernoulli equation (Table 1). Change in mean pressure gradient was defined as the difference between the pressure gradient values measured pre-procedurally and during follow-up.

\section{Endpoint definition for adverse events}

Clinical, imaging, and laboratory data were evaluated at follow-up study visits by a cardiologist at the same day of $\mathrm{CT}$ and echocardiographic 


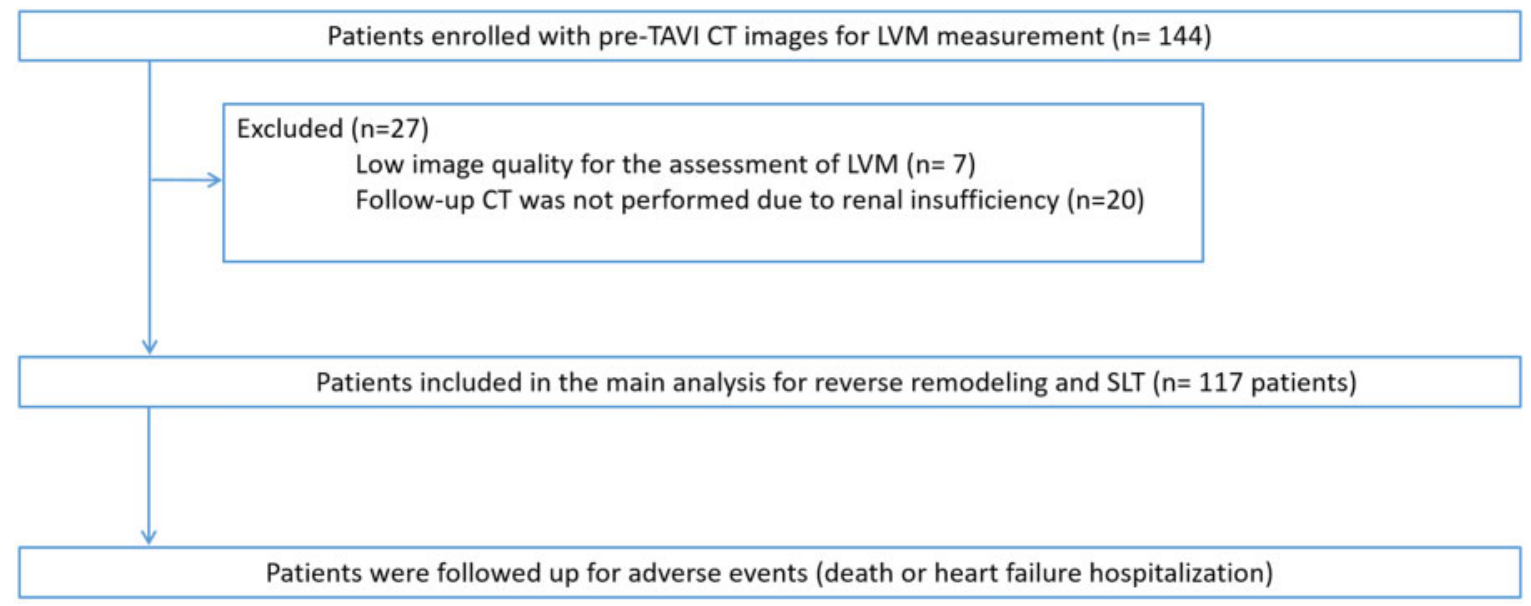

Figure I Study flowchart.

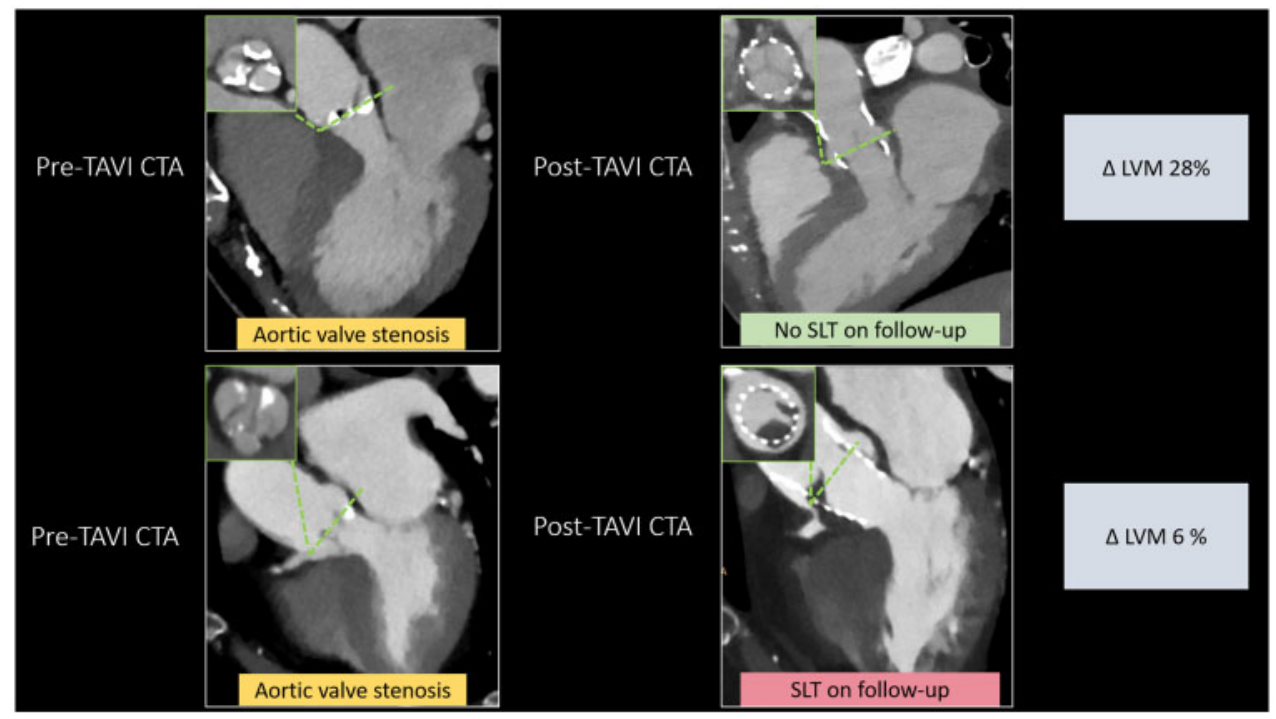

Figure 2 Representative cases for the assessment of reverse remodelling and SLT. CTA images were analysed to quantify LVM using a semi-automated software. Valve assessment was performed by three radiologists on the follow-up images. LVM was measured on pre- and post-TAVI images to calculate change in LVM $(\Delta \mathrm{LVM})$. Case 1 (upper panels) depicts a patient without SLT and a prominent reverse remodelling, whereas Case 2 (lower panels) shows a patient with SLT and a reduced degree of reverse remodelling (see cross-sections).

imaging. Definition of hospitalization for heart failure included any hospitalization event that lasted for $>24 \mathrm{~h}$ during the follow-up period, at which patient demonstrated at least two heart failure-related symptoms (i.e. dyspnoea and oedema) and required intra venous diuretic therapy. ${ }^{21}$ Mortality data were collected and verified via official death records of the National Health Insurance Fund, which ensured that no patient was lost during the follow-up period. Mean follow-up time was 2.6 years. Composite endpoint was defined as all-cause mortality or heart failure hospitalization during follow-up, survival time was calculated from the date of TAVI to the date of confirmed death, hospitalization or last contact with the patient. We compared clinical outcomes in patients with more and less than $20 \%$ reduction in LV mass. ${ }^{22}$

\section{Statistical analysis}

Continuous variables are presented as mean and standard deviation, categorical parameters are presented as frequency with percentages. Continuous clinical and imaging variables between baseline and follow-up were compared using Wilcoxon signed-rank test. Categorical variables were compared using the $\chi^{2}$ test. Univariate and multivariate logistic 


\section{Table I Patient characteristics}

\begin{tabular}{lc}
\hline Parameters, $\boldsymbol{n}=\mathbf{1 1 7}$ & $54(46.2)$ \\
Male, $n$ (\%) & $79.0 \pm 7.5$ \\
Age, years & $26.9 \pm 4.7$ \\
Body mass index, $\mathrm{kg} / \mathrm{m}^{2}$ & $1.8 \pm 0.2$ \\
Body surface area, $\mathrm{m}^{2}$ & \\
Cardiovascular risk factors & $115(98.3)$ \\
Hypertension, $n$ (\%) & $34(29.1)$ \\
Diabetes mellitus, $n$ (\%) & $92(78.6)$ \\
Hyperlipidaemia, $n$ (\%) & $40(34.2)$ \\
Smoking in history, $n$ (\%) & $29(24.8)$ \\
Prior myocardial infarction, $n$ (\%) & \\
Echocardiographic parameters & $52.2 \pm 14.6 \ldots \ldots \ldots \ldots \ldots \ldots$ \\
Mean aortic transvalvular gradient (pre-TAVI) $\mathrm{mmHg}$ & $8.8 \pm 4.6$ \\
Mean aortic transvalvular gradient (follow-up) $\mathrm{mmHg}$ & $85.9 \pm 23.1$ \\
Peak aortic transvalvular gradient (pre-TAVI) $\mathrm{mmHg}$ & $16.7 \pm 8.3$ \\
Peak aortic transvalvular gradient (follow-up) $\mathrm{mmHg}$ & $43.4 \pm 15.2$ \\
Change in mean aortic transvalvular gradient & $69.1 \pm 24.4$ \\
Change in peak aortic transvalvular gradient & $84(71.8)$ \\
Medication & $94(80.3)$ \\
Statin therapy, $n$ (\%) & $90(76.9)$ \\
ACE-l/ARB therapy, $n$ (\%) & $36(30.8)$ \\
Beta-blocker therapy, $n$ (\%) & $36(30.8)$ \\
Oral anticoagulant therapy, $n$ (\%) & \\
Dual antiplatelet therapy, $n$ (\%) &
\end{tabular}

Continuous variables are presented as mean $\pm \mathrm{SD}$; categorical parameters are presented as frequencies and percentages. Change in mean and peak aortic transvalvular gradient was calculated as the difference between the pressure gradient values measured pre-TAVI and at follow-up.

$A C E-I$, angiotensin-converting-enzyme inhibitor; ARB, angiotensin II receptor blockers.

regression analyses were performed to detect the association between LV reverse remodelling and clinical parameters, echocardiographic parameters, cardiovascular risk factors, and SLT. Cox proportional hazard regression models were derived to assess the prognostic value of LV reverse remodelling. Kaplan-Meier curves were generated for the composite endpoint of hospitalization for heart failure and all-cause mortality. A $P$-value $<0.05$ was considered statistically significant. All calculations were performed using SPSS software (SPSS version 25; IBM Corp., Armonk, NY, USA).

\section{Results}

In total, 144 patients were enrolled in our study. Seven patients were excluded due to inadequate image quality (lower contrast accumulation in the right ventricle). In 20 cases, follow-up CT could not be performed due to impaired renal function, therefore, these cases were not included in the analysis. A total of 234 CTAs of 117 patients (mean age $79.0 \pm 7.5$ years, $53.8 \%$ female) were included in the final analysis (Figure 1). In total, 24.8\% (29/117) of the patients had prior myocardial infarction, 98.3\% (115/117) had hypertension, and 78.6\% (92/117) had hyperlipidaemia. Oral anticoagulant medication was administered in $30.8 \%$ (36/117) of the patients due to atrial fibrillation. SLT was reported in $25.6 \%(30 / 117)$ cases. The number of

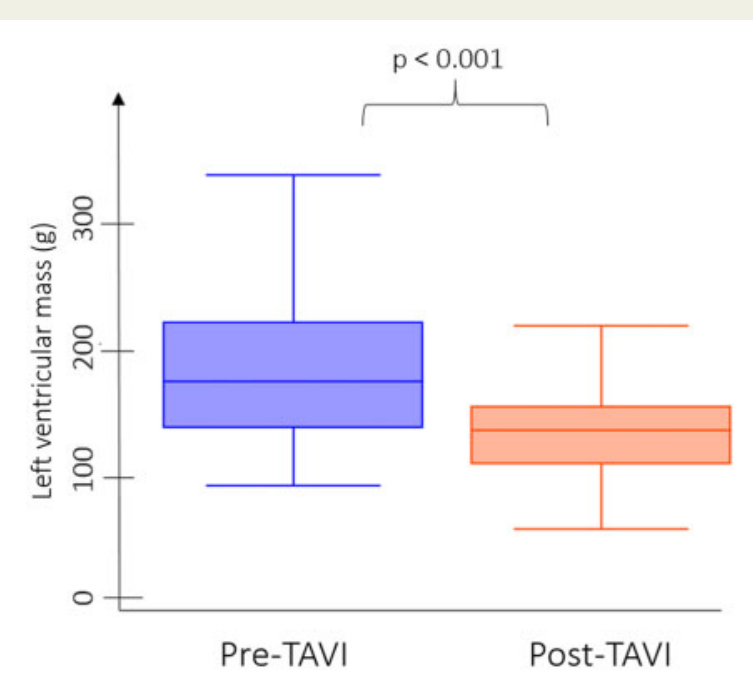

Figure 3 Boxplots demonstrating the impact of TAVI on LVM.

leaflets affected by SLT was evaluated, 20 patients were diagnosed with 1 cusp involvement, 9 patients with 2 cusps, and 1 patient with all 3 leaflets involved with SLT. Ejection fraction (EF) on TTE was $57.9 \pm 14.1 \%$ for pre-TAVI and $58.0 \pm 9.3 \%$ at follow-up. EF on follow-up echocardiography was $55.1 \pm 7.8 \%$ in patients with SLT and $59.0 \pm 9.5 \%$ in patients without SLT $(P=0.052)$. Also, EF on follow-up imaging was $59.0 \pm 7.9 \%$ and $55.0 \pm 10.5 \%$ for patient with and without LV reverse remodelling, respectively $(P<0.01)$. Mean aortic transvalvular gradient before TAVI was $52.2 \pm 14.6 \mathrm{mmHg}$ vs $8.8 \pm 4.6 \mathrm{mmHg}$ at follow-up $(P<0.01)$. Patient characteristics and imaging parameters are summarized in Table 1 . The mean radiation dose (dose-length product) was $772.7 \pm 541.0 \mathrm{mGy} \times \mathrm{cm}$.

\section{LV reverse remodelling following TAVI}

LVM and LVM index decreased substantially following TAVI $\left(180.5 \pm 53.0\right.$ vs. $137.1 \pm 44.8 \mathrm{~g}$ and $99.7 \pm 25.4$ vs. $75.4 \pm 19.9 \mathrm{~g} / \mathrm{m}^{2}$ for pre-TAVI and follow-up, respectively, both $P<0.001$; Figure 3 ). Overall, an average $43.4( \pm 33.9) \mathrm{g}$ of LVM reduction was detected at follow-up. Ten patients did not exhibit a reduction in LVM, whereas $>20 \%$ reduction in LVM was found in $62.4 \%$ patients $(73 / 117)$. Patients with lesser LV reverse remodelling had significantly higher prevalence of prior myocardial infarction and SLT (all $P<0.05$, Supplementary data online, Table S1). We detected significantly larger reduction in transaortic mean and peak pressure gradient values at follow-up among patients with reverse remodelling as compared with those without (both $P<0.05$, Supplementary data online, Table S1).

\section{Predictors of LV reverse remodelling}

In order to identify potential predictors of LV reverse remodelling, the association of various clinical and imaging parameters was investigated (see Table 2). Age, gender, cardiovascular risk factors, anticoagulant therapy did not show any association with reverse remodelling (all $P>0.05$ ). On the other hand, prior myocardial infarction $(P=0.002)$, the change in mean aortic transvalvular pressure gradient $(P=0.002)$ and the presence of SLT $(P=0.025)$ showed a 
Table 2 Predictors of LV reverse remodelling following TAVI

\begin{tabular}{|c|c|c|c|c|}
\hline \multirow[t]{2}{*}{ Parameters } & \multicolumn{2}{|c|}{ Univariate model } & \multicolumn{2}{|c|}{ Multivariate model } \\
\hline & OR & $P$-value & OR & $P$-value \\
\hline ACE-I/ARB therapy & 0.57 & 0.311 & & \\
\hline Myocardial infarction in history & 0.28 & 0.002 & 0.22 & 0.006 \\
\hline SLT & 0.35 & 0.025 & 0.27 & 0.022 \\
\hline Atrial fibrillation & 0.74 & 0.497 & & \\
\hline Oral anticoagulant therapy & 0.79 & 0.599 & & \\
\hline Change in mean aortic transvalvular pressure gradient (10 mmHg change) & 1.52 & 0.002 & 1.51 & 0.004 \\
\hline
\end{tabular}

Parameters with significant association with LV reverse remodelling on univariate regression were entered into a multivariate model and also the model was also corrected for age, gender, and following risk factors: hypertension, BMI, diabetes mellitus and hyperlipidaemia.

ACE-I, angiotensin-converting-enzyme inhibitor; ARB, angiotensin II receptor blockers; OR, odds ratio; SLT, subclinical leaflet thrombosis.

significant association with LV reverse remodelling after TAVI in univariate analysis. In multivariate logistic regression analysis, we found that SLT was inversely and independently associated with LV remodelling over age, gender, prior myocardial infarction change in mean pressure gradient and traditional risk factors (hypertension, dyslipidaemia, body mass index, and diabetes mellitus); OR 0.27, $P=0.022$ (Table 2). The number of leaflets affected by SLT did not show an association with reverse remodelling $(P=0.391)$.

\section{The prognostic relevance of $L V$ reverse remodelling}

During a mean follow-up time of 2.6 years, 13 adverse events occurred including 9 deaths and 4 heart failure hospitalizations. Patients with $>20 \%$ reduction in their myocardial mass following TAVI showed longer event-free survival as compared with those with a lower degree of reverse remodelling (Figure 4). LVM reduction of $>20 \%$ remained an independent predictor of event-free survival after adjustment for significant covariates in univariate analysis (Table 3).

\section{Discussion}

We demonstrated that TAVI leads to substantial LVM reduction. Our results suggest that the presence of SLT might be risk factor for impaired LV reverse remodelling. In addition, we found that impaired $L V$ reverse remodelling is associated with adverse clinical outcomes.

CT holds the potential to non-invasively detect early changes of implanted bioprosthetic valves. Currently, there are no guidelines on the diagnostic work-up of patients with suspected SLT, although experts of the field recommend regular echocardiographic surveillance to identify patients with elevated pressure gradient, thickened cusps or restricted cusp mobility as possible candidates for subsequent CT imaging. ${ }^{23,24}$ Importantly, there is also no consensus or unified criteria for SLT assessment on CT images and a variety of methods are in use currently across providers. ${ }^{25,26}$ In our study, the prevalence of SLT was $25.6 \%$, which is in line with previous studies. ${ }^{27}$ Our study complements the results of other reports regarding SLT and LV mass change after prosthetic valve implantation and shows a strong association between valve changes and reverse remodelling.

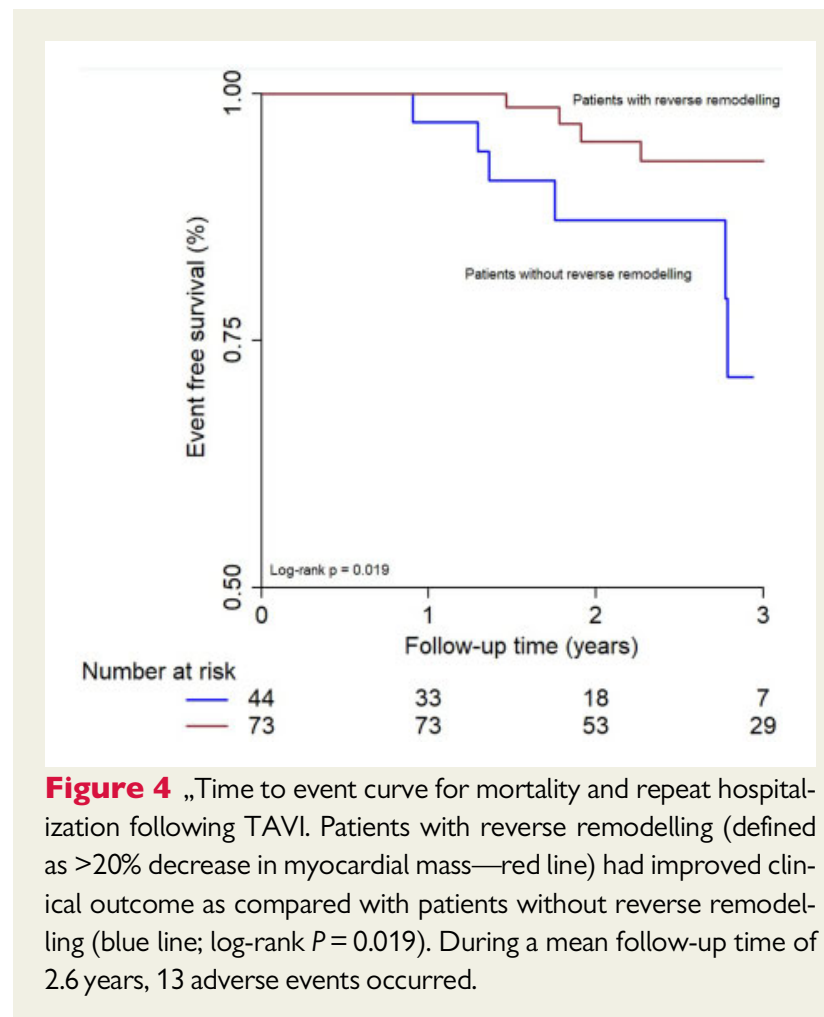

Our study aimed to provide evidence on the interplay of SLT and reverse remodelling to decipher the importance of LV structural changes in patients treated with TAVI utilizing CTA. Importantly, patients who developed SLT had reduced reverse remodelling. Magalhaes et al. ${ }^{28}$ analysed a cohort of 333 patients following TAVI and aimed to assess the normalization of LV geometry using TTE. The authors demonstrated that reverse remodelling process is not completed even at 1 year after TAVI. Very recent studies using cardiac magnetic resonance imaging (CMR) demonstrated that detectable reverse remodelling occurs as soon as within days after TAVI, ${ }^{29,30}$ suggesting that LV recovery is - in some cases-a rapid process and the largest observable reduction in LVM develops during the first few weeks following normalization of transaortic flow/ 
Table 3 Cox regression models to define independent predictors of events

\begin{tabular}{|c|c|c|c|c|}
\hline & \multicolumn{2}{|c|}{ Univariate model } & \multicolumn{2}{|c|}{ Multivariate model } \\
\hline & HR & $P$-value & HR & $P$-value \\
\hline Age & 1.07 & 0.149 & & \\
\hline Body mass index & 1.01 & 0.925 & & \\
\hline Diabetes mellitus & 4.13 & 0.016 & 3.57 & 0.031 \\
\hline Hypertension & 20.63 & 0.763 & & \\
\hline ACE-I/ARB therapy & 0.68 & 0.591 & & \\
\hline Myocardial infarction in history & 1.54 & 0.481 & & \\
\hline Reverse remodelling $>20 \%$ & 0.23 & 0.019 & 0.27 & 0.033 \\
\hline SLT & 0.87 & 0.829 & & \\
\hline Atrial fibrillation & 1.36 & 0.604 & & \\
\hline Oral anticoagulant therapy & 1.56 & 0.445 & & \\
\hline Change in mean aortic transvalvular pressure gradient & 0.964 & 0.102 & & \\
\hline
\end{tabular}

ACE-l, angiotensin-converting-enzyme inhibitor; ARB, angiotensin II receptor blockers; SLT, subclinical leaflet thrombosis.

pressure gradient. Subsequently, there is a trend for a slower improvement that can last up to a $12-24$ months. ${ }^{31}$ Similarly, studies suggest that the occurrence of SLT varies in time individually. ${ }^{23}$ SLT can develop within weeks (acute) or months (late) after TAVI, and progress over time without treatment leading to valve dysfunction and new-onset heart failure symptoms. Importantly, SLT can affect the function of the bioprosthesis and result in elevated transvalvular pressure gradient and thus might impact reverse remodelling. ${ }^{23,32}$ These observations are reflected by our results as well.

LV hypertrophy increases the risk of sudden cardiac death, heart failure, and adverse cardiac events by several proposed mechanisms including accelerated atherosclerosis, impaired pump function, adverse remodelling, and arrhythmias related to ischaemia. ${ }^{33,34}$ Once the afterload is normalized, a beneficial structural response can occur in the LV. ${ }^{4}$ The magnitude of changes shows individual differences and LV recovery can be influenced by several clinical and valverelated parameters. Our results and prior echocardiography or CMR studies have consistently demonstrated a significant regression in LVM after AVR. $^{30,35}$ In the study of Vizzardi et al., 22135 TAVI patients were evaluated using serial TTE imaging, and $>20 \%$ decrease in LVM was detected in $\sim 70 \%$ of the patients. Also, results from the PARTNER (Placement of Aortic Transcatheter Valves) registry demonstrated an average of $17 \%$ reduction of LVM 1 year after implantation. ${ }^{36}$ However, it is unknown which patients exhibit complete LV remodelling, whereas in others only partial resolution of LV hypertrophy occurs. However, TEE and CMR studies cannot incorporate SLT as a potentially important influencing factor of LV remodelling. We demonstrated that SLT is inversely associated with reverse remodelling and thus might reshape risk prediction in this patient population.

It has been suggested that reverse remodelling is associated with better outcomes after surgical valve implantation, therefore, finding the clinical and imaging predictors of LV recovery is crucial. Vizzardi et al. ${ }^{22}$ found that only baseline LVM value was an independent predictor of subsequent reverse changes. The severity of aortic regurgitation $^{37}$ or myocardial fibrosis was proposed as risk factors in other studies. ${ }^{31}$ It has been suggested that patients without myocardial fibrosis are more prone to early reverse remodelling that can provide long-term benefit. Our results suggest that patients who suffered myocardial infarction (assumingly developed fibrosis) - irrespectively of the presence of SLT-have lower rates of reduction in myocardial mass.

Currently, there are very limited data on the prognostic value of reverse remodelling after TAVI. We found that the presence of SLT is associated with diminished reverse remodelling. Furthermore, the impaired LV reverse remodelling is independently associated with the composite endpoint of death or heart failure hospitalization. Ali et al. ${ }^{7}$ investigated patients after surgical AVR using echocardiography and found that lower reduction in LVM was associated with poor long-term prognosis. Investigators of the PARTNER randomized trial have described that greater reduction in LVM leads to lower hospitalization rates, but no difference in all-cause mortality was observed after 1 year, ${ }^{35}$ although this study had shorter follow-up. Our results are in agreement with the above findings, showing that larger LVM regression after valve replacement could provide long-term benefit by reducing repeat hospitalization for heart failure and all-cause mortality. More importantly, SLT and reverse remodelling are proposed as novel imaging targets for post-TAVI patient management.

A number of limitations of the present study must be acknowledged. The lack of unified diagnostic criteria for SLT makes comparisons between relevant studies difficult. Despite this, the SLT incidence in our population seems to be in line with previously published data. ${ }^{16,38}$ As reverse remodelling is a long process and SLT may develop at various time points, the optimal timing for imaging is currently unknown. We performed post-TAVI imaging at various time points. Also, reduced leaflet motion was not evaluated in our study, we used a criterion for SLT that identifies more severe forms of the disease, which is more likely cause obstruction and possibly reduced leaflet motion. In addition, the evaluation of reduced leaflet motion with CT is even more challenging than SLT assessment due to the limited temporal resolution of current CT scanners as compared with echocardiography. Despite a long mean follow-up time, for the 
composite endpoint a relatively low event rate was observed and allcause mortality was used. Nonetheless, our study provides the first evidence of the impact of reverse remodelling on adverse events using CT imaging. Long-term outcome studies are warranted to guide clinical practice on the diagnosis and management of SLT.

In conclusion, our study demonstrates the favourable long-term effects of TAVI on LV morphology using serial CT imaging. Our study provides insight into the interplay between SLT, reverse remodelling and clinical outcomes. We found that the presence of SLT of the bioprosthetic valve and prior myocardial infarction might inhibit reverse remodelling process. Moreover, reverse remodelling following TAVI is associated with improved clinical outcome.

\section{Supplementary data}

Supplementary data are available at European Heart Journal - Cardiovascular Imaging online.

\section{Funding}

Project no. NVKP_16-1-2016-0017 ('National Heart Program') has been implemented with the support provided from the National Research, Development and Innovation Fund of Hungary, financed under the NVKP_16 funding scheme. The research was financed by the Higher Education Institutional Excellence Programme of the Ministry for Innovation and Technology in Hungary, within the framework of the Therapeutic Development thematic programme of the Semmelweis University. A.I.N. was supported by the János Bolyai Scholarship of the Hungarian Academy of Sciences. P.M.-H. was supported by the ÚNKP 2017/2018 Grant.

\section{Conflict of interest: none declared.}

\section{References}

1. lung B, Baron G, Butchart EG, Delahaye F, Gohlke-Barwolf C, Levang OW et al. A prospective survey of patients with valvular heart disease in Europe: the Euro Heart Survey on Valvular Heart Disease. Eur Heart J 2003;24:1231-43.

2. Heuvelman HJ, van Geldorp MW, Eijkemans MJ, Rajamannan NM, Bogers AJ, Roos-Hesselink JW et al. Progression of aortic valve stenosis in adults: a systematic review. J Heart Valve Dis 2012;21:454-62.

3. Rassi AN, Pibarot P, Elmariah S. Left ventricular remodelling in aortic stenosis. Can J Cardiol 2014;30:1004-11.

4. Treibel TA, Kozor R, Schofield R, Benedetti G, Fontana M, Bhuva AN et al. Reverse myocardial remodeling following valve replacement in patients with aortic stenosis. J Am Coll Cardiol 2018;71:860-71.

5. van Rosendael PJ, Delgado V, Bax JJ. Pacemaker implantation rate after transcatheter aortic valve implantation with early and new-generation devices: a systematic review. Eur Heart J 2018;39:2003-13.

6. Genereux P, Head SJ, Wood DA, Kodali SK, Williams MR, Paradis JM et al. Transcatheter aortic valve implantation 10-year anniversary: review of current evidence and clinical implications. Eur Heart J 2012;33:2388-98.

7. Ali A, Patel A, Ali Z, Abu-Omar Y, Saeed A, Athanasiou T et al. Enhanced left ventricular mass regression after aortic valve replacement in patients with aortic stenosis is associated with improved long-term survival. J Thorac Cardiovasc Surg 2011;142:285-91.

8. Vizzardi E, Sciatti E, Bonadei I, Rovetta R, D'Aloia A, Gelsomino S et al. Effects of transcatheter aortic valve implantation on left ventricular mass and global longitudinal strain: tissue Doppler and strain evaluation. Heart Lung Vessel 2014;6: 253-61.

9. Spethmann S, Dreger H, Baldenhofer G, Stuer K, Saghabalyan D, Muller E et al. Short-term effects of transcatheter aortic valve implantation on left atrial mechanics and left ventricular diastolic function. J Am Soc Echocardiogr 2013;26: 64-71.e2.

10. Kamperidis V, Joyce E, Debonnaire P, Katsanos S, van Rosendael PJ, van der Kley $\mathrm{F}$ et al. Left ventricular functional recovery and remodeling in low-flow low- gradient severe aortic stenosis after transcatheter aortic valve implantation. J Am Soc Echocardiogr 2014;27:817-25.

11. Spethmann S, Baldenhofer G, Dreger H, Stuer K, Sanad W, Saghabalyan D et al. Recovery of left ventricular and left atrial mechanics in various entities of aortic stenosis 12 months after TAVI. Eur Heart J Cardiovasc Imaging 2014;15:389-98.

12. Marwick TH, Gillebert TC, Aurigemma G, Chirinos J, Derumeaux G, Galderisi M et al. Recommendations on the use of echocardiography in adult hypertension: a report from the European Association of Cardiovascular Imaging (EACVI) and the American Society of Echocardiography (ASE)dagger. Eur Heart J Cardiovasc Imaging 2015;16:577-605.

13. Lang RM, Badano LP, Mor-Avi V, Afilalo J, Armstrong A, Ernande L et al. Recommendations for cardiac chamber quantification by echocardiography in adults: an update from the American Society of Echocardiography and the European Association of Cardiovascular Imaging. Eur Heart J Cardiovasc Imaging 2015;16:233-70.

14. Foppa M, Duncan BB, Rohde LE. Echocardiography-based left ventricular mass estimation. How should we define hypertrophy? Cardiovasc Ultrasound 2005;3:17.

15. Maffei E, Messalli G, Martini C, Nieman K, Catalano O, Rossi A et al. Left and right ventricle assessment with Cardiac CT: validation study vs. Cardiac MR. Eur Radiol 2012;22:1041-9.

16. Vollema EM, Kong WKF, Katsanos S, Kamperidis V, van Rosendael PJ, van der Kley $\mathrm{F}$ et al. Transcatheter aortic valve thrombosis: the relation between hypoattenuated leaflet thickening, abnormal valve haemodynamics, and stroke. Eur Heart J 2017;38:1207-17.

17. Delgado V, Ng AC, van de Veire NR, van der Kley F, Schuijf JD, Tops LF et al. Transcatheter aortic valve implantation: role of multi-detector row computed tomography to evaluate prosthesis positioning and deployment in relation to valve function. Eur Heart J 2010;31:1114-23.

18. Ng AC, Delgado V, van der Kley F, Shanks M, van de Veire NR, Bertini M et al. Comparison of aortic root dimensions and geometries before and after transcatheter aortic valve implantation by 2- and 3-dimensional transesophageal echocardiography and multislice computed tomography. Circ Cardiovasc Imaging 2010; 3:94-102.

19. Blanke P, Schoepf UJ, Leipsic JA. CT in transcatheter aortic valve replacement. Radiology 2013;269:650-69.

20. Pache G, Schoechlin S, Blanke P, Dorfs S, Jander N, Arepalli CD et al. Early hypo-attenuated leaflet thickening in balloon-expandable transcatheter aortic heart valves. Eur Heart J 2016;37:2263-71.

21. Zannad F, Stough WG, Pitt B, Cleland JG, Adams KF, Geller NL et al. Heart failure as an endpoint in heart failure and non-heart failure cardiovascular clinical trials: the need for a consensus definition. Eur Heart J 2008;29:413-21.

22. Vizzardi E, D’Aloia A, Fiorina C, Bugatti S, Parrinello G, De Carlo M et al. Early regression of left ventricular mass associated with diastolic improvement after transcatheter aortic valve implantation. J Am Soc Echocardiogr 2012;25:1091-8.

23. Dangas GD, Weitz Jl, Giustino G, Makkar R, Mehran R. Prosthetic heart valve thrombosis. J Am Coll Cardiol 2016;68:2670-89.

24. Spartera M, Ancona F, Barletta M, Rosa I, Stella S, Marini C et al. Echocardiographic features of post-transcatheter aortic valve implantation thrombosis and endocarditis. Echocardiography 2018;35:337-45.

25. Ruile P, Minners J, Breitbart P, Schoechlin S, Gick M, Pache G et al. Medium-term follow-up of early leaflet thrombosis after transcatheter aortic valve replacement. JACC Cardiovasc Interv 2018;11:1164-71.

26. Ruile P, Minners J, Schoechlin S, Pache G, Hochholzer W, Blanke P et al. Impact of the type of transcatheter heart valve on the incidence of early subclinical leaflet thrombosis. Eur J Cardiothorac Surg 2018;53:778-83.

27. Sondergaard L, De Backer O, Kofoed KF, Jilaihawi H, Fuchs A, Chakravarty T et al. Natural history of subclinical leaflet thrombosis affecting motion in bioprosthetic aortic valves. Eur Heart J 2017;38:2201-7.

28. Magalhaes MA, Koifman E, Torguson R, Minha S, Gai J, Kiramijyan S et al. Outcome of left-sided cardiac remodeling in severe aortic stenosis patients undergoing transcatheter aortic valve implantation. Am J Cardiol 2015;116: 595-603.

29. Dobson LE, Musa TA, Uddin A, Fairbairn TA, Swoboda PP, Erhayiem B et al. Acute reverse remodelling after transcatheter aortic valve implantation: a link between myocardial fibrosis and left ventricular mass regression. Can J Cardiol 2016;32:1411-8

30. Nucifora G, Tantiongco JP, Crouch G, Bennetts J, Sinhal A, Tully PJ et al. Changes of left ventricular mechanics after trans-catheter aortic valve implantation and surgical aortic valve replacement for severe aortic stenosis: a tissuetracking cardiac magnetic resonance study. Int J Cardiol 2017;228:184-90.

31. Fairbairn TA, Steadman CD, Mather AN, Motwani M, Blackman DJ, Plein S et al. Assessment of valve haemodynamics, reverse ventricular remodelling and myocardial fibrosis following transcatheter aortic valve implantation compared to surgical aortic valve replacement: a cardiovascular magnetic resonance study. Heart 2013;99:1185-91. 
32. Franzone A, Pilgrim T, Haynes AG, Lanz J, Asami M, Praz F et al. Transcatheter aortic valve thrombosis: incidence, clinical presentation and long-term outcomes. Eur Heart J Cardiovasc Imaging 2018;19:398-404.

33. Koren MJ, Devereux RB, Casale PN, Savage DD, Laragh JH. Relation of left ventricular mass and geometry to morbidity and mortality in uncomplicated essential hypertension. Ann Intern Med 1991;114:345-52.

34. Levy D. Clinical significance of left ventricular hypertrophy: insights from the Framingham Study. J Cardiovasc Pharmacol 1991;17(Suppl. 2):S1-6.

35. Lindman BR, Stewart WJ, Pibarot P, Hahn RT, Otto CM, Xu K et al. Early regression of severe left ventricular hypertrophy after transcatheter aortic valve replacement is associated with decreased hospitalizations. JACC Cardiovasc Interv 2014;7:662-73.
36. Athappan G, Patvardhan E, Tuzcu EM, Svensson LG, Lemos PA, Fraccaro C et al. Incidence, predictors, and outcomes of aortic regurgitation after transcatheter aortic valve replacement: meta-analysis and systematic review of literature. J Am Coll Cardiol 2013;61:1585-95.

37. Abdelghani M, Tateishi H, Miyazaki Y, Cavalcante R, Soliman Oll, Tijssen JG et al. Angiographic assessment of aortic regurgitation by video-densitometry in the setting of TAVI: echocardiographic and clinical correlates. Catheter Cardiovasc Interv 2017;90:650-9.

38. Marwan M, Mekkhala N, Goller M, Rother J, Bittner D, Schuhbaeck A et al. Leaflet thrombosis following transcatheter aortic valve implantation. J Cardiovasc Comput Tomogr 2018;12:8-13. 